\title{
Melioidosis after Hurricanes Irma and Maria, St. Thomas/St. John District, US Virgin Islands, October 2017
}

\section{Irene Guendel, ${ }^{1}$ Lisa LaPlace Ekpo, ${ }^{1}$ Mary K. Hinkle, Cosme J. Harrison, David D. Blaney, Jay E. Gee, Mindy G. Elrod, Sandra Boyd, Christopher A. Gulvik, Lindy Liu, Alex R. Hoffmaster, Brett R. Ellis, Tai Hunte-Ceasar, Esther M. Ellis}

We report 2 cases of melioidosis in women with diabetes admitted to an emergency department in the US Virgin Islands during October 2017. These cases emerged after Hurricanes Irma and Maria and did not have a definitively identified source. Poor outcomes were observed when septicemia and pulmonary involvement were present.

$\mathrm{M}$ elioidosis is caused by Burkholderia pseudomallei, a saprophytic, gram-negative bacillus endemic to tropical regions worldwide (1). Diagnosis is difficult because of wide-ranging clinical manifestations (2), and this bacterium is innately resistant to many antimicrobial drugs, making treatment options limited, complex, and lengthy (3). Infection occurs by percutaneous exposure, inhalation, or ingestion.

Melioidosis is rare in the United States, and cases are usually travel related $(4,5)$. However, regional endemicity has been documented in Puerto Rico (6), and sporadic human cases have been reported in the Caribbean (5,7). In September 2017, the US Virgin Islands were affected by 2 category 5 hurricanes, Irma and Maria; widespread flooding continued for weeks. We describe the clinical manifestations, management, and outcome of posthurricane melioidosis cases in 2 women in St. Thomas and St. John, US Virgin Islands.

\section{The Study}

Despite major damage to the 2 hospitals in the territory during the 2 hurricanes, the Virgin Islands Department of Health (VIDOH) maintained surveillance at both emergency

Author affiliations: Virgin Islands Department of Health, St. Thomas, Virgin Islands, USA (I. Guendel, L. LaPlace Ekpo, C.J. Harrison, B.R. Ellis, T. Hunte-Ceasar, E.M. Ellis); Walter Reed Army Institute of Research, Silver Spring, Maryland, USA (M.K. Hinkle); Centers for Disease Control and Prevention, Atlanta, Georgia, USA (D.D. Blaney, J.E. Gee, M.G. Elrod, S. Boyd, C.A. Gulvik, L. Liu, A.R. Hoffmaster) departments. Two isolates were recovered from each patient. Local specimen analysis for organism identification was performed by using the MicroScan WalkAway System (Siemens Healthcare Diagnostics, https://www.siemenshealthineers.com). All isolates were confirmed as B. pseudomallei at the Centers for Disease Control and Prevention (CDC; Atlanta, GA, USA). Whole-genome sequencing and single-nucleotide polymorphism analysis were performed (National Center for Biotechnology Information, https:// www.ncbi.nlm.nih.gov, Project PRJNA488733). Genomes from a given patient were clonal to each other. However, representative genomes from both patients had differences $(>5,600$ single-nucleotide polymorphisms), indicating the presence of different strains in these infections. Genomic comparison with a reference panel indicated that the isolates were within the previously described Western Hemisphere clade and subclade associated with the Caribbean (8).

Patient 1 was an 80-year-old female resident of St. Thomas who had a history of cardiomyopathy and type II diabetes mellitus. She came to the emergency department (ED) at Schneider Regional Medical Center (St. Thomas, US Virgin Islands) because of shortness of breath (symptom onset 28 days after Hurricane Irma and 9 days after Hurricane Maria). Her symptoms were worsened orthopnea, increased abdominal girth, and edema, consistent with her symptoms at previous admissions. The patient was admitted for management of acute decompensated heart failure.

The patient had a temperature of $98.5^{\circ} \mathrm{F}$; diffuse pulmonary crackles; jugular venous distension; normal heart sounds; and bilateral, lower extremity pitting edema. Examination showed a focal area on the anterior left thigh that had a central, firm, warm, erythematous, tender, subcutaneous nodule $\approx 2 \mathrm{~cm}$ in diameter with a central fluctuant area and a small pinhole. Incision and drainage was performed, and a swab specimen of purulent drainage was sent for culture.

The patient was given intravenous clindamycin $(600$ mg every $8 \mathrm{~h}$ for $5 \mathrm{~d}$ ) and was discharged while receiving oral clindamycin, but the treatment course was not completed. Cultured wound showed growth of B. pseudomallei at $\approx 5$ days. However, culture growth was not yet positive before patient discharge. The isolate was susceptible to trimethoprim/sulfamethoxazole (Table 1).

${ }^{1}$ These authors contributed equally to this article. 
Table 1. Culture results and antimicrobial drug susceptibility for Burkholderia pseudomallei isolated from 2 case-patients with melioidosis after Hurricanes Irma and Maria, St. Thomas/St. John District, US Virgin Islands, October 2017*

\begin{tabular}{|c|c|c|c|c|c|}
\hline Patient, culture & Drug & $\begin{array}{l}\text { MicroScan Walk Away } \\
\text { System MIC, } \mu \mathrm{g} / \mathrm{mL}\end{array}$ & Result & $\mathrm{CDC} \mathrm{MIC}, \mu \mathrm{g} / \mathrm{mL}$ & Result \\
\hline \multirow[t]{7}{*}{1 , first wound culture } & Amoxicillin/clavulanate & $\mathrm{NT}$ & NA & $4 / 2$ & $\mathrm{~S}$ \\
\hline & Ceftazidime & $>16$ & $\mathrm{R}$ & 4 & $\mathrm{~S}$ \\
\hline & Doxycycline & NT & NA & 1 & $\mathrm{~S}$ \\
\hline & Imipenem & NT & NA & 0.5 & $S$ \\
\hline & Tetracycline & NT & NA & 4 & $S$ \\
\hline & Trimethoprim/sulfamethoxazole & $\leq 2 / 38$ & $S$ & $\leq 0.5 / 9.5$ & S \\
\hline & Meropenem & NT & NA & -1 & $\ddagger$ \\
\hline \multirow[t]{7}{*}{ 1, second wound culture } & Amoxicillin/clavulanate & NT & NA & $4 / 2$ & $\mathrm{~S}$ \\
\hline & Ceftazidime & $>16$ & $\mathrm{R}$ & 2 & S \\
\hline & Doxycycline & NT & NA & 1 & $\mathrm{~S}$ \\
\hline & Imipenem & NT & NA & 0.5 & $\mathrm{~S}$ \\
\hline & Tetracycline & NT & NA & 4 & S \\
\hline & Trimethoprim/sulfamethoxazole & $\leq 2 / 38$ & $S$ & $\leq 0.5 / 9.5$ & $\mathrm{~S}$ \\
\hline & Meropenem & NT & NA & -1 & $\ddagger$ \\
\hline \multirow[t]{7}{*}{2 , sputum culture } & Amoxicillin/clavulanate & NT & NA & $4 / 2$ & $\mathrm{~S}$ \\
\hline & Ceftazidime & 4 & $\mathrm{~S}$ & 4 & $\mathrm{~S}$ \\
\hline & Doxycycline & NT & NA & 1 & $\mathrm{~S}$ \\
\hline & Imipenem & NT & NA & 0.5 & $\mathrm{~S}$ \\
\hline & Tetracycline & NT & NA & 4 & $\mathrm{~S}$ \\
\hline & Trimethoprim/sulfamethoxazole & $\leq 2 / 38$ & S & $\leq 0.5 / 9.5$ & $\mathrm{~S}$ \\
\hline & Meropenem & NT & NA & 1 & $\ddagger$ \\
\hline
\end{tabular}

${ }^{*} \mathrm{CDC}$, Centers for Disease Control and Prevention; NA, not applicable; NT, not tested; R, resistant; S, susceptible.

†Siemens Healthcare Diagnostics, https://www.siemens-healthineers.com.

$\ddagger$ There are no published breakpoints in the Clinical and Laboratory Standards Institute M45 (9).

Patient 1 returned to the ED 2 weeks later because of manifestations similar to those at the first visit. She was afebrile and admitted for diuresis. The left thigh lesion had progressed into a $2-\mathrm{cm}$, tender, shallow ulcer productive of purulent material surrounded by erythema and a focal area of induration (Figure). Laboratory data reflected a leukocyte count within reference ranges and mild renal insufficiency with estimated glomerular filtration rate of 40.47 $\mathrm{mL} / \mathrm{min}$ (Table 2).

A second wound culture was collected, and the patient was given intravenous meropenem ( $1 \mathrm{~g}$ every $8 \mathrm{~h})$. Culture was presumptively positive for $B$. pseudomallei and Serratia marcescens after $\approx 48$ hours, confirmed after 8 days. Both isolates showed the same resistance pattern and were susceptible to meropenem and trimethoprim/sulfamethoxazole: the MIC for meropenem was $<1 \mu \mathrm{g} / \mathrm{mL}$ (Table 2). Meropenem was continued for 8 days, and ulcer improvement was observed. The patient was discharged while receiving oral trimethoprim/sulfamethoxazole $(800 \mathrm{mg} / 160$ $\mathrm{mg} 2 \times / \mathrm{d}$ ) to complete maintenance therapy. The patient completed a 3-month course of trimethoprim/sulfamethoxazole and achieved resolution.

Patient 2 was a 60 -year-old female who was a resident of St. John who had diabetes. She was referred to the ED at Schneider Regional Medical Center by her primary care physician because of hyperglycemia, productive cough, and malaise for 1 week (symptom onset 46 days after Hurricane Irma and 33 days after Hurricane Maria). The patient was admitted to the intensive care unit because of community-acquired pneumonia.
The patient was lethargic and had a temperature of $101^{\circ} \mathrm{F}$; heart rate was 99 beats/min, respiratory rate 22 breaths/min, and blood pressure 142/81 mm Hg. Blood gas

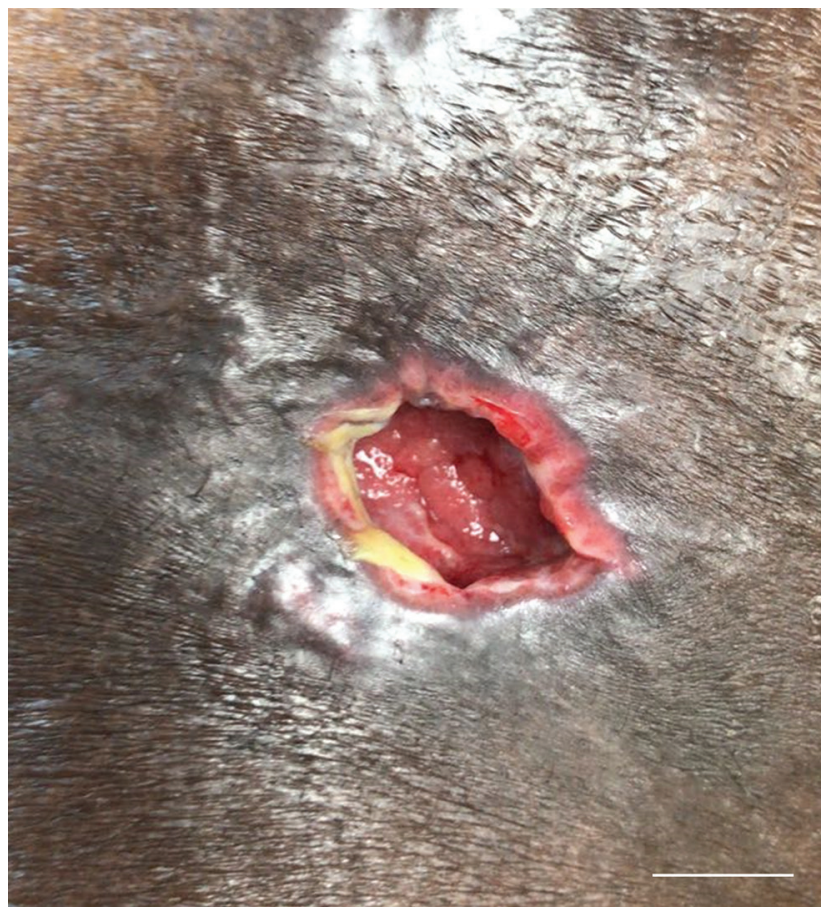

Figure. Cutaneous melioidosis lesion in case-patient 1 after Hurricanes Irma and Maria, St. Thomas/St. John District, US Virgin Islands, October 2017. This lesion was on the left anterior thigh and had a diameter of $2 \mathrm{~cm}$. Shown is a tender, shallow, ulcer productive of purulent material surrounded by erythema and a focal area of induration. Scale bar indicates $1 \mathrm{~cm}$. 
Table 2. Laboratory values for 2 case-patients with melioidosis after Hurricanes Irma and Maria, St. Thomas/St. John District, US Virgin Islands, October, $2017^{*}$

\begin{tabular}{|c|c|c|c|c|c|c|c|}
\hline \multirow[b]{2}{*}{ Parameter } & \multicolumn{3}{|c|}{ Patient 1} & \multicolumn{3}{|c|}{ Patient 2} & \multirow[b]{2}{*}{ Reference range } \\
\hline & Oct 18 & Oct 21 & Oct 24 & Oct 26 & Oct 27 & Oct 30 & \\
\hline Leukocytes & 4.2 & NT & 4.1 & 28.3 & 18.1 & 12.6 & $4.8-10.8 \times 10^{3} / \mathrm{mm}^{3}$ \\
\hline Hemoglobin B & 11.9 & NT & 15.5 & 11.3 & 10.4 & 8.1 & $12.0-14.0 \mathrm{~g} / \mathrm{L}$ \\
\hline Hematocrit & 38.1 & NT & 48.9 & 34.6 & 31.5 & 24.0 & $36.0-42.0 \%$ \\
\hline Platelets & 185 & NT & 174 & 441 & 345 & 201 & $140-440 \times 10^{3} / \mathrm{mm}^{3}$ \\
\hline Neutrophils & 67.0 & NT & 46.2 & 92.5 & 89.5 & 92.6 & $40.0 \%-75.0 \%$ \\
\hline Lymphocytes & 20.8 & NT & 38.2 & 1.8 & 2.4 & 5.1 & $15.0 \%-45.5 \%$ \\
\hline Monocytes & 9.6 & NT & 10.8 & 4.9 & 5.3 & 2.2 & $0.0 \%-10.0 \%$ \\
\hline Eosinophils & 0.6 & NT & 3.7 & 0.7 & 2.7 & 0.0 & $0.0 \%-6.0 \%$ \\
\hline Basophils & 2.0 & NT & 1.1 & 0.1 & 0.1 & 0.1 & $0.0 \%-2.0 \%$ \\
\hline Sodium & 134 & 127 & NT & 125 & 130 & 137 & $136-145 \mathrm{mmol} / \mathrm{L}$ \\
\hline Potassium & 4.8 & 3.6 & NT & 3.5 & 2.9 & 3.2 & $3.6-5.2 \mathrm{mmol} / \mathrm{L}$ \\
\hline Chloride & 100 & 91 & NT & 87 & 95 & 104 & 98-107 mmol/L \\
\hline Bicarbonate & 28.0 & 31.3 & NT & 17.5 & 21.5 & 16.5 & $21-32 \mathrm{mmol} / \mathrm{L}$ \\
\hline Blood urea nitrogen & 23 & 18 & NT & 17 & 17 & 63 & 7-18 mg/dL \\
\hline Creatinine & 1.58 & 1.26 & NT & 1.19 & 1.07 & 3.92 & $0.6-1.3 \mathrm{mg} / \mathrm{dL}$ \\
\hline Glucose & 169 & 213 & NT & 367 & 235 & 404 & $70-110 \mathrm{mg} / \mathrm{dL}$ \\
\hline Hemoglobin A1C & NT & NT & NT & NT & NT & 11 & $4.5 \%-6.2 \%$ \\
\hline Calcium & 8.2 & 9.1 & NT & 10.2 & 9.0 & 8.3 & 8.5-10.5 mg/dL \\
\hline Phosphorus & NT & 3.3 & NT & NT & NT & 1.9 & $2.4-4.9 \mathrm{mg} / \mathrm{dL}$ \\
\hline Magnesium & NT & NT & NT & NT & NT & 1.9 & $1.8-2.4 \mathrm{mg} / \mathrm{dL}$ \\
\hline Total bilirubin & 0.6 & NT & NT & 1.5 & 1.5 & 2.3 & $0.0-1.0 \mathrm{mg} / \mathrm{dL}$ \\
\hline Direct bilirubin & NT & NT & NT & NT & NT & 2.0 & $0.0-0.3 \mathrm{md} / \mathrm{dL}$ \\
\hline AST & 32 & NT & NT & 34 & 52 & 49 & 15-37 U/L \\
\hline ALT & 27 & NT & NT & 25 & 25 & 34 & 12-78 U/L \\
\hline Alkaline phosphatase & 94 & NT & NT & 155 & 138 & 142 & 50-136 U/L \\
\hline Total protein & 7.2 & NT & NT & 8.0 & 6.4 & 5.1 & $6.4-8.2 \mathrm{~g} / \mathrm{dL}$ \\
\hline Albumin & 3.10 & 2.70 & NT & 2.10 & 1.6 & 0.8 & $3.4-5.0 \mathrm{~g} / \mathrm{dL}$ \\
\hline
\end{tabular}

testing showed $\mathrm{pO} 2$ of $47.6 \mathrm{~mm} \mathrm{Hg}$ with an oxygen saturation of $87.2 \%$ on 2-liter nasal cannula. A chest radiograph showed a left-sided mild infiltrate, and her leukocyte count was markedly increased $\left(28.3 \times 10^{3}\right.$ cells $\left./ \mathrm{mm}^{3}\right)($ Table 2$)$.

The patient was given intravenous ceftriaxone $(1 \mathrm{~g} / \mathrm{d})$ and azithromycin $(500 \mathrm{mg} / \mathrm{d})$ after blood and sputum cultures were prepared. She required bilevel positive airway pressure but eventually required mechanical ventilation. The patient then became hypotensive and required norepinephrine to maintain a main arterial pressure $\geq 65 \mathrm{~mm} \mathrm{Hg}$. Ceftriaxone was discontinued, and she was given intravenous piperacillin/tazobactam (3.375 g every $6 \mathrm{~h})$. Trimethoprim/sulfamethoxazole- and ceftazidime-sensitive $B$. pseudomallei were identified from sputum culture after 72 hours (Table 1). Methicillin-sensitive Staphylococcus aureus and Candida glabrata were also identified. One of 2 blood cultures was positive for gram-negative rods. Piperacillin/tazobactam was discontinued, and the patient was given meropenem ( $1 \mathrm{~g}$ every $8 \mathrm{~h})$.

The patient remained critically ill and was transferred to a tertiary care hospital in the continental United States. She died in a long-term care facility during October 2018 without showing signs of neurologic improvement.

Isolates from both patients showed susceptibility to routinely tested antimicrobial drugs $(10,11)$. Isolates from patient 1 showed resistance to ceftazidime during preliminary analysis (Table 1). However, broth microdilution confirmatory testing performed at CDC indicated ceftazidime susceptibility, highlighting the need for additional antimicrobial resistance confirmation.

Both patients were interviewed to determine travel history and possible exposure sources. Patient 1 traveled occasionally to the southeastern United States; her last travel date was 3 months before her illness. This patient reported flooding and water damage to her home from the hurricanes, but did not report contact with flood waters. Patient 2 reported no travel history before the hurricanes.

VIDOH has investigated and confirmed a subsequent case-patient with pulmonary melioidosis in St. Thomas during December 2018 (I. Guendel et al., unpub. data). This case-patient reported no recent travel and might have had occupational exposure as a professional gardener. This person had 2 risk factors (type II diabetes mellitus and heavy use of alcohol).

\section{Conclusions}

Given regional occurrence, detection of melioidosis in the US Virgin Islands is not surprising. Furthermore, emergence of melioidosis after extreme weather events has been well documented, and cases were likely acquired locally from storm-related exposure to flooded soil, surface water runoff, or generation of coarse aerosols $(12,13)$. Although detection of $B$. pseudomallei has yet to be confirmed in the environment, it might be endemic to the US Virgin Islands, as in Puerto Rico. 
In January 2018, melioidosis was listed as a reportable disease in the US Virgin Islands. Future actions include disease education efforts for physicians and laboratory staff because misdiagnosis is common (14). Awareness campaigns highlighting preventive measures for the public are necessary because risk factors are prevalent in the local population (e.g., diabetes and other chronic disease) and might be exacerbated under disaster settings (e.g., respiratory effects and open wounds). VIDOH has implemented rapid diagnostic testing by using Active Melioidosis Detect (InBios International, https://inbios.com) on suspected specimens for prompt on-island case identification while routine ED diagnostic cultures are performed (5). All confirmatory testing is conducted at CDC.

\section{Acknowledgments}

We thank Beulah Pinney, LaToya Harrigan, Maritza Phillip, and infection control staff from Schneider Regional Medical Center for providing assistance.

This study was supported by the Epidemiology and Laboratory Capacity for Infectious Diseases Cooperative Agreement from the Centers for Disease Control and Prevention.

\section{About the Author}

Dr. Guendel is an epidemiologist at the US Virgin Islands Department of Health. Her research interests are emerging and remerging infectious diseases.

\section{References}

1. Wiersinga WJ, Currie BJ, Peacock SJ. Melioidosis. N Engl J Med. 2012;367:1035-44. https://doi.org/10.1056/NEJMra1204699

2. Cheng AC, Currie BJ. Melioidosis: epidemiology, pathophysiology, and management. Clin Microbiol Rev. 2005;18:383-416. https://doi.org/10.1128/CMR.18.2.383-416.2005

3. Wuthiekanun V, Amornchai P, Saiprom N, Chantratita N, Chierakul W, Koh GC, et al. Survey of antimicrobial resistance in clinical Burkholderia pseudomallei isolates over two decades in northeast Thailand. Antimicrob Agents Chemother. 2011;55:538891. https://doi.org/10.1128/AAC.05517-11

4. Stewart T, Engelthaler DM, Blaney DD, Tuanyok A, Wangsness E, Smith TL, et al. Epidemiology and investigation of melioidosis, southern Arizona. Emerg Infect Dis. 2011;17:1286-8. https://doi.org/10.3201/eid1707.100661

5. Benoit TJ, Blaney DD, Doker TJ, Gee JE, Elrod MG, Rolim DB, et al. A review of melioidosis cases in the Americas. Am J Trop Med Hyg. 2015;93:1134-9. https://doi.org/10.4269/ajtmh.15-0405

6. Doker TJ, Sharp TM, Rivera-Garcia B, Perez-Padilla J, Benoit TJ, Ellis EM, et al. Contact investigation of melioidosis cases reveals regional endemicity in Puerto Rico. Clin Infect Dis. 2015;60:24350. https://doi.org/10.1093/cid/ciu764

7. Sanchez-Villamil JI, Torres AG. Melioidosis in Mexico, Central America, and the Caribbean. Trop Med Infect Dis. 2018;3:pii:24.

8. Gee JE, Gulvik CA, Elrod MG, Batra D, Rowe LA, Sheth M, et al. Phylogeography of Burkholderia pseudomallei isolates, Western Hemisphere. Emerg Infect Dis. 2017;23:1133-8. https://doi.org/10.3201/eid2307.161978

9. Clinical and Laboratory Standards Institute. Methods for antimicrobial dilution and disk susceptibility testing of infrequently isolated or fastidious bacteria. 3rd ed (M45). Wayne (PA): The Institute; 2016.

10. Limmathurotsakul D, Peacock SJ. Melioidosis: a clinical overview. Br Med Bull. 2011;99:125-39. https://doi.org/10.1093/bmb/ldr007

11. Wuthiekanun V, Peacock SJ. Management of melioidosis. Expert Rev Anti Infect Ther. 2006;4:445-55. https://doi.org/10.1586/ 14787210.4.3.445

12. Cheng AC, Jacups SP, Gal D, Mayo M, Currie BJ. Extreme weather events and environmental contamination are associated with case-clusters of melioidosis in the Northern Territory of Australia. Int J Epidemiol. 2006;35:323-9. https://doi.org/10.1093/ ije/dyi271

13. Merritt AJ, Inglis TJ. The role of climate in the epidemiology of melioidosis. Curr Trop Med Rep. 2017;4:185-91. https://doi.org/ 10.1007/s40475-017-0124-4

14. Hemarajata P, Baghdadi JD, Hoffman R, Humphries RM. Burkholderia pseudomallei: challenges for the clinical microbiology laboratory. J Clin Microbiol. 2016;54:2866-73. https://doi.org/10.1128/JCM.01636-16

Address for correspondence: Esther M. Ellis, Virgin Islands Department of Health, 1303 Hospital Ground Rd, Ste 10, VI 00802, USA; email: esther.ellis@doh.vi.gov 